\title{
Evaluación del lipidograma y ciertos factores de riesgo de ateroesclerosis en niños cuyos padres tuvieron arteriopatía coronaria de inicio temprano
}

\author{
Assessment of lipid profile and some risk factors of atherosclerosis in children whose \\ parents had early onset coronary artery disease
}

\begin{abstract}
Dra.Helen Bornaun ${ }^{a}$, Prof.Dr. Naci Öner ${ }^{b}$,Prof.Dr.Kemal Nişlic, Dr. Kazım Öztarhan ${ }^{a}$, Prof. Asoc.Dr.Taner Yavuz, Prof. Dra. Ümit Türkoğluc , Prof. Dra. Aygün Dindarc y Prof.Dra. Rukiye Eker Ömeroglu
\end{abstract}

\section{RESUMEN}

Antecedentes/Objetivo. El objetivo de nuestro estudio fue analizar el lipidograma y ciertos factores de riesgo de ateroesclerosis, tales como las lipoproteínas de baja densidad oxidadas (ox-LDL, por su sigla en inglés) y las lipoproteínas de baja densidad pequeñas y densas (sdLDL, por su sigla en inglés) en los hijos de pacientes con cardiopatía coronaria (CC) prematura.

Población y métodos. Hijos de padres con CC deinicio temprano emparejados con pares de su misma edad y mismo sexo. Se analizaron las concentraciones de lípidos, apolipoproteínas (ApoA, B, E), ox-LDL, sdLDL y lipoproteína (a) [Lp(a)] en los niños de estudio y de referencia. Los datos se evaluaron con el programa SPSS, junto con la prueba t de Student y la prueba U de Mann-Whitney.

Resultados. Los niños del grupo deestudio (n: 43) tenían niveles más elevados de LDL, Lp(a) y ox-LDL y cocientes mayores de CT/HDL, ApoB/ApoA, LDL/HDL y ox-LDL/HDL $(p<0,05)$ que los del grupo de referencia.

Conclusión. Con base en estos hallazgos, se sugiere que la dislipidemia y las concentraciones elevadas de LDL, Lp(a) y ox-LDL son frecuentes en los hijos de pacientes con CC de inicio temprano y representan gran parte de la predisposición familiar a tener CC.

Palabras clave. Arteriopatía coronaria, lípidos, hijos, factores de riesgo, niños.

http:/ / dx.doi.org/10.5546/aap.2017.50

Texto completo en inglés:

http://dx.doi.org/10.5546/aap.2017.eng.50

a. Clínica de Cardiología Pediátrica, Hospital Universitario y de Investigación Sultan Süleyman, Estambul, Turquía.

b. Departamento de Cardiología Pediátrica, Yeni Yüzyil Üniversitesi, Istambul, Turquía.

c. Departamento de Cardiología Pediátrica, Facultad de Medicina de Istanbul Üniversitesi, Estambul, Turquía.

d. Departamento de Cardiología Pediátrica, Hospital Universitario y de Investigación Zeynep Kamil, Estambul, Turquía.

Correspondencia: Prof. Dr. C. Naci Öner: nacioner@yahoo.com

Financiamiento: este estudio recibió financiación de la

Fundación de Investigaciones de la Istanbul Üniversitesi.

Conflicto de intereses: Ninguno que declarar.

Recibido: 28-4-2016.

Aceptado: 11-8-2016.

\section{INTRODUCCIÓN}

La ateroesclerosis se define como una enfermedad que comienza en las primeras etapas de la vida y que produce cardiopatía coronaria (CC) en la madurez y posteriormente. Los familiares de primer grado y los hijos de los pacientes que tuvieron CC antes de los 55 años de edad tienen un riesgo mayor de tener CC. ${ }^{1,2}$

El proceso se inicia mediante la captura y oxidación de las lipoproteínas en los lugares propensos a la formación de lesiones. Se ha demostrado que las lipoproteínas de baja densidad (LDL, por su sigla en inglés) son citotóxicas y definitivamente aterogénicas debido a la modificación oxidativa en los cultivos de células endoteliales. Se considera que las LDL oxidadas (ox-LDL, por su sigla en inglés) conforman el nuevo parámetro de valor predictivo en el desarrollo de ateroesclerosis. ${ }^{2}$

Las LDL tienen una estructura heterogénea y están compuestas por dos fenotipos de partículas de tamaños diferentes. El tipo A es más grande y contiene partículas de LDL más flotantes. El tipo B es más pequeño y contiene partículas de LDL pequeñas y densas (sdLDL, por su sigla en inglés). Las sdLDL tienen propiedades aterogénicas potentes debido a la mayor penetración de la pared arterial, la menor tendencia de fijación a los receptores de LDL, la semivida prolongada en plasma y, probablemente, la mayor sensibilidad a los cambios oxidativos.

Varios estudios transversales demostraron asociaciones significativas entre el tamaño de las partículas de LDL, especialmente las concentraciones de sdLDL, y la CC. ${ }^{3}$ En muchos estudios, la función predictiva de las apolipoproteínas (reducción de la ApoA y ApoE y aumento de la ApoB) y de la lipoproteína (a) [Lp(a)] en el desarrollo de la CC es más relevante que el de los lípidos séricos. ${ }^{4,5}$ El objetivo de nuestro estudio fue analizar el lipidograma y ciertos factores de riesgo de ateroesclerosis, tales como las ox-LDL, las sdLDL y la Lp(a) en los hijos de los pacientes con CC prematura. 


\section{POBLACIÓN Y MÉTODOS}

Este estudio prospectivo se llevó a cabo en el Departamento de Cardiología Pediátrica de la Facultad de Medicina de Estambul entre 2010 y 2015, con la autorización del Comité de Ética local. El grupo de estudio estaba compuesto por niños sanos de entre 6 y 18 años de edad, hijos de personas que tuvieron CC prematura (hombres $<45$ años, mujeres $<55$ años). El grupo de referencia estaba compuesto por niños sanos de la misma edad y el mismo sexo que no tenían antecedentes familiares de CC prematura. Todos los grupos se seleccionaron de los consultorios externos, donde se les había detectado un soplo funcional. Se excluyó del estudio a los niños que tenían deformación cardíaca, hipertensión o una enfermedad crónica, como diabetes mellitus, insuficiencia renal o hepática, desnutrición o un trastorno genético o endocrino que afectara el crecimiento y el desarrollo cardíaco. Se obtuvo el consentimiento informado de los padres de los niños que participaron en el estudio.

Se compararon los factores de riesgo de CC, el índice de masa corporal (IMC), y la presión arterial (PA) sistólica y diastólica entre ambos grupos. Los parámetros bioquímicos se evaluaron con muestras de sangre obtenidas en ayunas; los análisis de colesterol total (CT), triglicéridos (TG), lipoproteínas de alta densidad (HDL, por su sigla en inglés), Lp(a), ApoA y ApoB se hicieron con Integra-800 (Roche) conforme a la fórmula de Friedewald. Las concentraciones de ApoE se analizaron con un método nefelométrico con el sistema analizador automático BN ProSpec (Dade Behring). El método modificado de decantación de heparina-magnesio se usó para el análisis de sdLDL. El nivel de ox-LDL se midió con el método
ELISA (kit oLAB, Biomedica).

Los grupos se compararon con la prueba $t$ de Student y la prueba U de Mann-Whitney. Un valor de $p<0,05$ se consideró estadísticamente significativo.

\section{RESULTADOS}

Se emparejó a cuarenta y tres hijos de padres con CC de inicio temprano (grupo de estudio) con pares de su misma edad y mismo sexo (grupo de referencia, $n=43$ ). No se observaron diferencias estadísticamente significativas entre ambos grupos con respecto al peso, la estatura, el IMC, y la PA sistólica y diastólica (Tabla 1).

Se compararon los lipidogramas de ambos grupos. Los resultados de la comparación se presentan en la Tabla 2. No se observaron diferencias estadísticamente significativas en relación con los valores de CT, TG, VLDL y sdLDL. Las concentraciones de LDL, Lp(a) y ox-LDL fueron más elevadas en el grupo de estudio en comparación con el de referencia $(p<0,05)$ (Tabla 2). No se observaron diferencias estadísticamente significativas en relación con el cociente de TG/HDL y LDL/ApoB entre los grupos. En la comparación entre grupos se establecieron diferencias estadísticamente significativas respecto al cociente de LDL/HDL, CT/HDL, ApoB/ApoA y ox-LDL/HDL (Tabla 2).

\section{DISCUSIÓN}

Este estudio es el primero en investigar los factores de riesgo lipídicos de CC en los hijos de pacientes con CC prematura en la población turca. Según nuestros datos, esta población tiene concentraciones significativamente elevadas de LDL, ox-LDL y Lp(a). Dada la edad de los casos,

TABLA 1. Características del grupo de estudio y el grupo de referencia

\begin{tabular}{lccc}
\hline & $\begin{array}{c}\text { Grupo de estudio } \\
\mathbf{n}=\mathbf{4 3}\end{array}$ & $\begin{array}{c}\text { Grupo de referencia } \\
\mathbf{n}=4 \mathbf{3}\end{array}$ & $p$ \\
\hline Edad (años) & $13,3 \pm 3,6$ & $13,3 \pm 3,6$ & - \\
& $(5,0-18,2 ; 14,0)$ & $(5,0-18,2 ; 14,0)$ & 0,41 \\
\hline Peso (kg) & $50,2 \pm 15,9$ & $50,7 \pm 17,4$ & $(15,4-85,2 ; 51,0)$ \\
& $(17,0-86,2 ; 49,0)$ & $1,5 \pm 0,2$ & 0,48 \\
\hline Estatura (m) & $1,5 \pm 0,2$ & $(1,03-1,79 ; 1,59)$ & 0,91 \\
& $(1,06-1,79 ; 1,59)$ & $20,6 \pm 4,1$ & \\
\hline IMC (kg/m $)$ & $21,0 \pm 4,1$ & $(12,8-30,5 ; 21,6)$ & 0,53 \\
& $(13,5-30,8 ; 21,2)$ & $115,8 \pm 10,0$ & $(89-138 ; 120)$ \\
\hline Presión arterial sistólica (mmHg) & $115,3 \pm 12,2$ & $70,1 \pm 8,1$ & $(54-85 ; 75)$ \\
\hline Presión arterial diastólica $(\mathbf{m m H g})$ & $(89-146 ; 114)$ & 0,67 \\
\hline
\end{tabular}

Los datos se presentan como media \pm desviación estándar (mínimo-máximo, mediana).

IMC: índice de masa corporal. 
un motivo relevante para tal predisposición podría atribuirse a los factores genéticos. ${ }^{1,2}$

La disfunción endotelial es una etapa muy temprana de la ateroesclerosis, que es una de las manifestaciones patológicas más frecuentes de la enfermedad vascular. La interacción entre las lipoproteínas y las células endoteliales es crítica en la generación y el desarrollo de la ateroesclerosis. Las ox-LDL fomentan la patogenia y el desarrollo de la ateroesclerosis, y la proliferación, migración y alteración fenotípica de las células musculares lisas vasculares, que las convierte en macrófagos espumosos, son cambios críticos en esta enfermedad. También tendrían una función novedosa en la patología. ${ }^{3}$
Johnston y col., ${ }^{6}$ concluyeron que las concentraciones de ox-LDL y el cociente de oxLDL/HDL constituyen una medición superior y más precisa en comparación con los lipidogramas clásicos habituales.

Sin embargo, la mayoría de estos estudios se llevaron a cabo en poblaciones adultas, y los estudios sobre los factores de riesgo aterogénicos en niños son aún limitados. De manera similar a nuestro estudio, Kelishadi y col., ${ }^{7}$ demostraron una elevación de los metabolitos de ox-LDL en los niños con antecedentes familiares de CC de inicio temprano.

En nuestro estudio también se reveló que el cociente de ox-LDL/HDL era más alto en el

TABLA 2. Comparación de los parámetros lipídicos en el grupo de estudio y el grupo de referencia

\begin{tabular}{|c|c|c|c|}
\hline & $\begin{array}{l}\text { Grupo de estudio } \\
\qquad n=43\end{array}$ & $\begin{array}{l}\text { Grupo de referencia } \\
\qquad n=43\end{array}$ & $p$ \\
\hline Colesterol total (CT) & $\begin{array}{c}138,2 \pm 27,6 \\
(64,2-97,4 ; 129,1)\end{array}$ & $\begin{array}{c}129,9 \pm 27,8 \\
(86,2-207,4 ; 132,1)\end{array}$ & NS \\
\hline Triglicéridos (TG) & $\begin{array}{c}96,2 \pm 58,7 \\
(26,2-281,2 ; 81,4)\end{array}$ & $\begin{array}{c}85,9 \pm 49,5 \\
(25,2-305,3 ; 84,4)\end{array}$ & NS \\
\hline HDL & $\begin{array}{c}41,6 \pm 13,1 \\
(10,4-79,1 ; 42,3)\end{array}$ & $\begin{array}{c}46,4 \pm 10,8 \\
(24,2-74,3 ; 44,4)\end{array}$ & NS \\
\hline LDL & $\begin{array}{c}77,6 \pm 18,9 \\
(36,4-110,1 ; 70,2)\end{array}$ & $\begin{array}{c}66,2 \pm 20,4 \\
(36,4-125,1 ; 72,0)\end{array}$ & $<0,01$ \\
\hline VLDL & $\begin{array}{c}19,2 \pm 11,7 \\
(5,0-56,1 ; 15,2)\end{array}$ & $\begin{array}{c}17,24 \pm 9,9 \\
(5,0-61,1 ; 16,2)\end{array}$ & NS \\
\hline Lp(a) & $\begin{array}{c}12,2 \pm 7,4 \\
(4,1-37,2 ; 9,5)\end{array}$ & $\begin{array}{c}9,1 \pm 5,1 \\
(2,1-22,2 ; 8,4)\end{array}$ & $<0,05$ \\
\hline ox-LDL & $\begin{array}{c}418,4 \pm 180,1 \\
(108,1-909,4 ; 342,0)\end{array}$ & $\begin{array}{c}302,0 \pm 162,9 \\
(100,1-792,4 ; 308,2)\end{array}$ & $<0,01$ \\
\hline sdLDL & $\begin{array}{c}11,0 \pm 4,7 \\
(1,4-22,7 ; 10,5)\end{array}$ & $\begin{array}{c}10,3 \pm 4,7 \\
(1,5-24,0 ; 10,5)\end{array}$ & NS \\
\hline ApoA & $\begin{array}{c}121,1 \pm 25,8 \\
(54,0-177,1 ; 120,4)\end{array}$ & $\begin{array}{c}129,1 \pm 22,2 \\
(70,4-170,2 ; 126,0)\end{array}$ & NS \\
\hline ApoB & $\begin{array}{c}57,8 \pm 13,2 \\
(22,2-84,2 ; 58,4)\end{array}$ & $\begin{array}{c}52,6 \pm 13,7 \\
(21,0-83,2 ; 54,3)\end{array}$ & NS \\
\hline ApoE & $\begin{array}{c}3,3 \pm 0,8 \\
(2,4-5,2 ; 2,7)\end{array}$ & $\begin{array}{c}4,60 \pm 7,2 \\
(2,4-5,1 ; 3,8)\end{array}$ & NS \\
\hline LDL/HDL & $\begin{array}{c}2,1 \pm 1,1 \\
(0,8-7,2 ; 1,8)\end{array}$ & $\begin{array}{c}1,5 \pm 0,5 \\
(0,8-3,1 ; 1,4)\end{array}$ & $<0,01$ \\
\hline TG/HDL & $\begin{array}{c}2,9 \pm 2,93 \\
(0,4-18,0 ; 1,8)\end{array}$ & $\begin{array}{c}2,1 \pm 1,9 \\
(0,5-12,0 ; 1,8)\end{array}$ & NS \\
\hline CT/HDL & $\begin{array}{c}3,7 \pm 1,6 \\
(1,9-11,8 ; 3,2)\end{array}$ & $\begin{array}{c}2,9 \pm 0,8 \\
(2,0-5,6 ; 2,9)\end{array}$ & $<0,01$ \\
\hline АроВ/ApoA & $\begin{array}{c}0,5 \pm 0,2 \\
(0,3-1,2 ; 0,5)\end{array}$ & $\begin{array}{c}0,4 \pm 0,1 \\
(0,4-1,4 ; 0,4)\end{array}$ & $<0,05$ \\
\hline LDL/ApoB & $\begin{array}{c}1,4 \pm 0,2 \\
(1,1-2,6 ; 1,4)\end{array}$ & $\begin{array}{c}1,3 \pm 0,3 \\
(0,8-2,5 ; 1,3)\end{array}$ & NS \\
\hline ox-LDL/HDL & $\begin{array}{c}11,3 \pm 7,1 \\
(2,5-44,4 ; 14.2)\end{array}$ & $\begin{array}{c}6,9 \pm 3,2 \\
(2,0-16,6 ; 7,2)\end{array}$ & $<0,05$ \\
\hline
\end{tabular}

Los datos se presentan como media \pm desviación estándar (mínimo-máximo, mediana).

HDL: lipoproteínas de alta densidad.

LDL: lipoproteínas de baja densidad.

VLDL: lipoproteínas de muy baja densidad.
Lp(a): lipoproteína-a. ox-LDL: LDL oxidadas. sdLDL: LDL pequeñas y densas. ApoA: apoliproteína A. ApoB: apoliproteína B. 
grupo de estudio. Con base en estos resultados, se concluye que las LDL son más sensibles a la oxidación en los niños con antecedentes familiares de CC de inicio temprano. Si estudios futuros respaldan nuestros hallazgos, los enfoques preventivos y los antioxidantes cobrarán relevancia, especialmente en los niños provenientes de familias con riesgo elevado. Además, el cociente de ox-LDL/HDL podría convertirse en una prioridad para la detección de los sujetos en riesgo, en lugar de utilizar los factores de riesgo familiar de CC típicos.

El cociente de los parámetros de lípidos y lipoproteínas respecto del riesgo de CC también se consideró importante en estos estudios. ${ }^{4,8}$ Los resultados de nuestro estudio concuerdan con los del estudio realizado por Widhalm y col., ${ }^{9}$ y Rallidis y col. ${ }^{10}$ Estos estudios demostraron una concentración más elevada de CT/HDL y LDL/HDL cuando estos cocientes de compararon entre ambos grupos.

La función predictiva de las apolipoproteínas en el desarrollo de la CC es superior al de los lípidos séricos. Beigel y col., ${ }^{11}$ indicaron que las apolipoproteínas representan un parámetro importante en la detección de los factores de riesgo coronario en los niños. Demostraron una concentración menor de ApoA, una concentración más elevada de $A$ poB y un cociente mayor de ApoB/ApoA en los sujetos de su estudio. Muchos investigadores ahora consideran que el cociente de ApoB/ApoA es un parámetro predictivo significativo para determinar los grupos en riesgo durante la evaluación de los factores de riesgo cardiovascular.

La Lp(a) está compuesta por una partícula central lipoprotéica, similar a las LDL, con la $A p o B$ unida mediante un enlace covalente a la glucoproteína ApoA, lo que produce el desarrollo de la placa ateroesclerótica. ${ }^{12,13}$ En nuestro estudio, demostramos que el grupo de estudio tenía valores más elevados de Lp(a). Rallidis ${ }^{10}$ y Wilcken y col., ${ }^{12}$ indicaron que el aumento de la $\mathrm{Lp}(\mathrm{a})$ era un factor de riesgo de CC importante en los niños con antecedentes familiares. Nuestro estudio también respalda el uso de los valores de Lp(a) como marcador aceptado para predefinir a los sujetos en riesgo entre los niños con antecedentes familiares de CC de inicio temprano.

En diversos estudios retrospectivos se demostraron asociaciones significativas entre las concentraciones de sdLDL y la CC. En los sujetos con un valor elevado de sdLDL, el riesgo de CC aumenta, independientemente de las concentraciones de LDL. La medición de este factor de riesgo podría ser relevante para la detección de los sujetos en riesgo desde la niñez. ${ }^{3}$ Según el estudio cardiovascular de Quebec, ${ }^{14}$ un aumento de la ApoB en las sdLDL es un factor de riesgo importante en el desarrollo de la CC de inicio temprano.

Algunos estudios recientes hallaron que el colesterol de las sdLDL tiene un valor predictivo para determinar qué sujetos tienen riesgo de CC. Por ejemplo, en un estudio realizado en Japón por Koba y col., ${ }^{15}$ en 2006, se observaron concentraciones mayores de sdLDL en los sujetos con CC en comparación con el grupo de referencia. En el mismo estudio se compararon los valores de sdLDL entre 225 sujetos de 45 a 75 años de edad con CC y el grupo de referencia, y se observó una diferencia significativa. Nuestros estudios no respaldan estos hallazgos, ya que los valores de sdLDL fueron similares en ambos grupos, lo cual podría atribuirse a la menor edad de nuestro grupo de estudio. ${ }^{3}$

La limitación más obvia de nuestro estudio fue el diseño basado en el hospital y el tamaño pequeño de la muestra. Por lo tanto, los resultados deben interpretarse con precaución. Otra limitación fue la evaluación de los sujetos en cuanto a la dieta y el ejercicio. Nuestro estudio incluyó solamente conversaciones con autoinforme y no se usó un cuestionario sobre dieta y ejercicio. Si bien este estudio tuvo limitaciones, puede aportar datos valiosos acerca de los niños cuyos padres hayan tenido CC de inicio temprano.

A modo de conclusión, los resultados de este estudio indican la necesidad de la detección temprana de la dislipidemia en los hijos de los sujetos con CC prematura. Estos niños podrían beneficiarse de medidas relacionadas con la alimentación y podrían establecerse actividades diarias como un primer paso hacia un estilo de vida saludable para la prevención primaria de la ateroesclerosis.

\section{REFERENCIAS}

1. Bao W, Srinivasan SR, Valdez R, Greenlud KJ, et al. Longitudinal changes in cardiovascular risk from childhood to young adulthood in offspring of parents with coronary artery disease: the Bogalusa Heart study. JAMA 1997;278(21):1749-54.

2. De Sutter JD, De Bacquer DD, Kotseva K, Sans S, et al. Screening of family members of patients with premature coronary heart disease; results from the EUROPASPIRE II family survey. Eur Heart J 2003;24(3):249-57.

3. Sumino H, Nakajima K, Murakami M. Possibility of New Circulating Atherosclerosis-Related Lipid Markers Measurement in Medical and Complete Medical Checkups: Small Dense Low-Density Lipoprotein Cholesterol and Lipoprotein Lipase. 2016;64(3):298-307.

4. Freedman DS, Srinivasan SR, Shear CL, Franklin FA, et al. The relation of apolipoproteins A-I and B in children to parental myocardial infarction. $N$ Engl J Med 1986;315(12):721-6.

5. Isser HS, Puri VK, Narain VS, Saran RK, et al. Lipoprotein (a) and Lipid levels in young patients with myocardial infarction and their first-degee relatives. Indian heart $J$ 2001;53(4):463-6. 
6. Johnston N, Jemberg T, Lagerrvist B, Siegbehn A, et al. Improved identification of patients with coronary artery disease by the use of new lipid and lipoprotein biomarkers. Am J Cardiol 2006;97(5):640-5.

7. Kelishadi R, Nadery GR, Asgary S. Oxidized LDL metabolites with high family risk for premature cardiovascular disease. Indian J Pediatr 2002;69(9):755-9.

8. Kelishadi R, Zadegan NS, Naderi GA, Asgary S, et al. Atherosclerosis risk factors in children and adolescents with or without family history of premature coronary artery disease. Med Sci Monit 2002;8(6):CR425-9.

9. Widhalm K, Koch S, Pakosta R, Schurz M, et al. Serum lipids, lipoproteins and apolipoprotein in children, with and without familial history of premature coronary heart disease. J Am Coll Nutr 1992;11(Suppl):S32-5.

10. Rallidis LS, Papageorgakis NH, Megalou AA, Exadactylos NJ, et al. High incidence of dyslipidaemia in the offspring of Greek men with premature coronary artery disease. Eur
Heart J 1998;19(3):395-401.

11. BeigelY, GeorgeJ,Leibovici L, Mattityahu A, etal.Coronary risk factors in children of parents with premature coronary artery disease. Acta Paediatr 1993;82(2):162-5.

12. Wilcken DE, Wang XL, Greenwood J, Lynch J. Lipoprotein(a) and apolipoproteins B and A-1 in children and coronary vascular events in their grandparents. J Pediatr 1993;123(4):519-26.

13. Ridker PM, Hennekes $\mathrm{CH}$, Stampfer MJ. A prospective study of Lipoprotein(a) and the risk of myocardial infarction. JAMA 1993;270(18):2195-9.

14. Brunzell JD. Increased Apo B in small Dense LDL particles predicts premature coronary artery disease. Arterioscler Thromb Vasc Biol 2005;25(3):474-5.

15. KobaS,Hirano T, Ito $Y$, Tsunoda F, etal.Significance of small dense low-density lipoprotein-cholesterol concentrations in relation to the severity of coronary heart diseases. Atherosclerosis 2006;189(1):206-14.

\title{
Saturación de oxígeno, respiración periódica y apnea durante el sueño en lactantes de 1 a 4 meses a 3200 metros de altura
}

\section{Oxygen saturation, periodic breathing, and sleep apnea in infants aged 1-4 months old living at 3200 meters above sea level}

\author{
Dr. Santiago Ucrós ${ }^{a}$ Dra. Claudia Granados ${ }^{b}$, Dra. Karem Parejoc , Dr. Fausto Ortega ${ }^{d}$, Dr. Fernando Guilléne, \\ Dra. Sonia Restrepof, M. Cs. Fabián Gill y Estud. Miriam Guillén ${ }^{h}$
}

a. Departamento de Pediatría. Fundación Santa Fe de Bogotá. Bogotá, Colombia.

b. Departamentos de Pediatría y Epidemiología Clínica y Bioestadística. Pontificia Universidad Javeriana. Bogotá, Colombia.

c. Departamento de Neurología y Laboratorio de Sueño. Fundación Clínica Shaio. Bogotá, Colombia.

d. Departamento de Pediatría. Hospital Luis Fernando Martínez. Cañar, Ecuador.

e. Departamento de Pediatría. Hospital del Río. Universidad del Azuay. Cuenca, Ecuador.

f. Departamentos de Pediatría. Hospital de La Misericordia y Fundación Santa Fe de Bogotá, Universidad de los Andes. Bogotá, Colombia.

g. Departamento de Epidemiología Clínica y Bioestadística. Pontificia Universidad Javeriana. Bogotá, Colombia.

h. Estudiante de Medicina. Universidad del Azuay. Cuenca, Ecuador.

Correspondencia:Dr. Santiago Ucrós, santiago_ucros@yahoo.com

Financiamiento: esta investigación fue parcialmente financiada con aportes de la Fundación Conocimiento, Bogotá, Colombia (GrantPI-FC/001-2012), el Fondo de Educación del Departamento de Pediatría de la Fundación Santa Fe de Bogotá y el Fondo para Investigación de la Universidad del Azuay, Cuenca, Ecuador.

Conflicto de intereses: Ninguno que declarar.

Recibido: 6-3-2016.

Aceptado: 12-8-2016

\section{RESUMEN}

Objetivos: describir, en niños de 1-4 meses, a $3200 \mathrm{~m}$ de altura, la saturación de oxígeno $\left(\mathrm{SpO}_{2}\right)$, los índices de apnea y la respiración periódica (RP) durante el sueño. Se realizaron polisomnografías en 18 lactantes sanos.

Resultados: las medianas fueron de $87 \%$ para la $\mathrm{SpO}_{2}$ y de $7,2 \%$ para la RP del tiempo total de sueño. El índice de apnea central tuvo una mediana de 30,5/hora, que disminuyó a 5,4/hora al descontar las apneas asociadas a RP. El p5 de la $\mathrm{SpO}_{2}$ para niños despiertos fue de $76 \%$ y, para niños dormidos, de $66 \%$. Conclusiones: la $\mathrm{SpO}_{2}$ fue inferior a la del nivel del mar y la RP y el índice de apnea central, mayores; al descontar las apneas centrales asociadas a RP. Este último, fue similar a la del nivel del mar. A $3200 \mathrm{~m}$, se requieren puntos diferentes para la $\mathrm{SpO}_{2}$ normal, uno para niños despiertos y otro si están dormidos. Palabras clave: sueño, lactante, altitud, oximetría, polisomnografía.

http:/ / dx.doi.org/10.5546/aap.2017.54

Texto completo en inglés:

http:/ /dx.doi.org/10.5546/aap.2017.eng.54

\section{INTRODUCCIÓN}

La saturación de oxígeno de la hemoglobina $\left(\mathrm{SpO}_{2}\right)$ en lactantes está establecida durante la vigilia a diferentes alturas. ${ }^{1-4}$ Sin embargo, los datos para niños durante el sueño son menos frecuentes. Al respecto, se han realizado estudios en Colombia, ${ }^{5-7}$ Bolivia, ${ }^{1}$ China, ${ }^{3}$ Estados Unidos ${ }^{4}$ y Argentina. ${ }^{8}$ No se encuentran disponibles publicaciones relacionadas con la $\mathrm{SpO}_{2}$ normal en lactantes de $1 \mathrm{a}$ 